\title{
Pre-clinical studies of a recombinant adenoviral mucosal vaccine to prevent $\mathrm{SARS-CoV-2}$ infection
}

Anne C. Moore ${ }^{1}$, Emery G. Dora ${ }^{2}$, Nadine Peinovich ${ }^{2}$, Kiersten P. Tucker ${ }^{2}$, Karen Lin², Mario Cortese $^{2}$, Sean N. Tucker ${ }^{2 *}$

${ }^{1}$ School of Biochemistry and Cell Biology, University College Cork, Cork, Ireland

${ }^{2}$ Vaxart, Inc. South San Francisco, CA, USA

*Corresponding author. Dr. Sean Tucker, Chief Scientist Officer, Vaxart, Inc. stucker@vaxart.com 


\section{SUMMARY}

There is an urgent need to develop efficacious vaccines against SARS-CoV-2 that also address the issues of deployment, equitable access, and vaccine acceptance. Ideally, the vaccine would prevent virus infection and transmission as well as preventing COVID-19 disease. We previously developed an oral adenovirus-based vaccine technology that induces both mucosal and systemic immunity in humans. Here we investigate the immunogenicity of a range of candidate adenovirusbased vaccines, expressing full or partial sequences of the spike and nucleocapsid proteins, in mice. We demonstrate that, compared to expression of the S1 domain or a stabilized spike antigen, the full length, wild-type spike antigen induces significantly higher neutralizing antibodies in the periphery and in the lungs, when the vaccine is administered mucosally. Antigen-specific CD4+ and CD8+ T cells were induced by this leading vaccine candidate at low and high doses. This fulllength spike antigen plus nucleocapsid adenovirus construct has been prioritized for further clinical development.

\section{INTRODUCTION}

The emergence of a novel coronavirus, severe acute respiratory syndrome coronavirus 2 (SARSCoV-2), the causative agent of COVID-19 disease, in 2019, has led to a global pandemic and significant morbidity, mortality and socio-economic disruption not seen in a century. Coronavirus disease 2019 (COVID-19) is a respiratory illness of variably severity; ranging from asymptomatic infection to mild infection, with fever and cough to severe pneumonia and acute respiratory distress $^{1}$. Current reports suggest that asymptomatic spread is substantial ${ }^{2}$, and SARS-CoV-2 infection induces a transient antibody response in most individuals ${ }^{3}$. Therefore, development of successful interventions is an immediate requirement to protect the global population against infection and transmission of this virus and its associated clinical and societal consequences. Mass immunization with efficacious vaccines has been highly successful to prevent the spread of many other infectious diseases and can also prevent disease in the vulnerable through the induction of herd immunity. Significant effort and resources are being invested in urgently identifying efficacious SARS-CoV-2 vaccines. A number of different vaccine platforms have demonstrated pre-clinical immunogenicity and efficacy against pneumonia ${ }^{4}, 5$. Several vaccines have demonstrated phase I or phase II safety and immunogenicity ${ }^{6-8}$. However, at this time, no vaccine has demonstrated efficacy in the field.

The most advanced SARS-CoV-2 vaccine candidates are all given by the intramuscular (IM) route, with some requiring $-80{ }^{\circ} \mathrm{C}$ storage. This is a major barrier for vaccine dissemination and deployment during a pandemic in which people are asked to practice social distancing and avoid congregation. The ultimate goal of any vaccine campaign is to protect against disease by providing enough herd immunity to inhibit viral spread, not to make a set number of doses of vaccine. An 
injected solution takes a long period of time to administer and distribute and requires costly logistics, which means dose availability does not immediately translate to immunity. Further, systemic immunization can induce immunity in the periphery and lower respiratory tract. However, these vaccines cannot induce mucosal immunity in the upper respiratory tract. Mucosal IgA (with the polymeric structure and addition of the secretory component), creates more potent viral neutralization ${ }^{9}$, can block viral transmission ${ }^{10,11}$, and in general, is more likely to create sterilizing immunity given that this is the first line of defense for a respiratory pathogen.

Mucosal vaccines can induce mucosal immune responses, antibodies and $\mathrm{T}$ cells at wet surfaces. We are developing oral vaccines for multiple indications, including influenza and noroviruses, delivered in a tablet form for people. Our vaccine platform is a replication-defective adenovirus type-5 vectored vaccine that expresses antigen along with a novel toll-like receptor 3 agonist as an adjuvant. These vaccines have been well tolerated, and able to generate robust humoral and cellular immune responses to the expressed antigens ${ }^{12-14}$. Protective efficacy in humans was demonstrated against a respiratory virus 90 days or more post vaccination, as shown in a well characterized experimental influenza infection mode ${ }^{15}$. Furthermore, the vaccine also has the advantage of room temperature stability and needle-free, ease of administration, providing several advantages over injected vaccine approaches with respect to vaccine deployment and access.

Here, we describe the pre-clinical development of a SARS-CoV-2 vaccine based on Vaxart's oral adenovirus platform. The key approach was to develop several vaccine candidates in parallel, in order to create premanufacturing seeds while initial immunogenicity experiments were in progress. Given that the vaccines were made during the pandemic, rapid decisions were required to keep the manufacturing and regulatory timelines from slipping. We assessed the relative immunogenicity of four candidate vaccines that expressed antigens based on the spike (S) and nucleocapsid (N) SARS-CoV-2 proteins. These proteins have been well characterized as antigens for related coronaviruses, such as SARS-CoV and MERS (reviewed in Yong, et al., ${ }^{16}$ ) and, increasingly, for SARS-CoV-2 spike. The aim of our vaccine is to induce immunogenicity on three levels; firstly, to induce potent serum neutralizing antibodies to $\mathrm{S}$, secondly to induce mucosal immune responses, and thirdly to induce $\mathrm{T}$ cell responses to both vaccine antigens. This three-fold approach aims to induce robust and broad immunity capable of protecting the individual from virus infection as well as disease, promote rapid dissemination of vaccine during a pandemic, and to protect the population from virus transmission through herd immunity.

Here, we report the induction of neutralizing antibody (Nab), IgG and IgA antibody responses, and $\mathrm{T}$ cell responses in mice following immunization of rAd vectors expressing one or more SARS-CoV-2 antigens.

\section{RESULTS}

\section{Vector Construction}

Initially, three different $r A d$ vectors were constructed to express different SARS-CoV-2 antigens. These were a vector expressing the full-length $\mathrm{S}$ protein (rAd-S), a vector expressing the S protein 
and the $\mathrm{N}$ protein (rAd-S-N), and a vector expressing a fusion protein of the $\mathrm{S} 1$ domain with the $\mathrm{N}$ protein (rAd-S1-N). The N protein of rAd-S-N was expressed under control of the human beta actin promoter, which is much more potent in human cells than mouse cells. An additional construct where the expressed S protein was fixed in a prefusion conformation (rAd-S(fixed)-N) was constructed at a later date as a control for exploring neutralizing antibody responses. These are described in Figure 1. Expression of the various transgenes was confirmed following infection of 293 cells using flow cytometry and monoclonal antibodies to the $\mathrm{S}$ or $\mathrm{N}$ protein (supplemental figure 1).

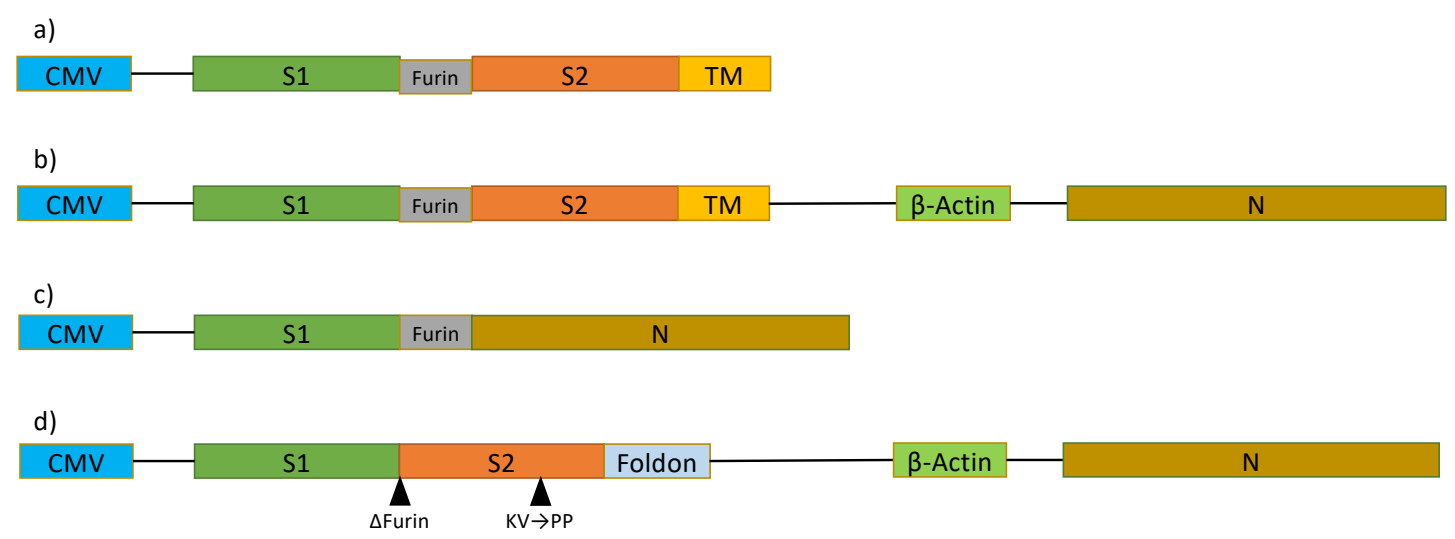

Figure 1. Transgene inserts developed to test vaccine specific responses. Recombinant adenoviruses were made using these inserts a. rAd-S b. rAd-S-N c.rAd-S1-N d. rAd-S(fixed)-N

\section{Immunogenicity of $\mathbf{r d d}$ vectors expressing $S$ and $\mathrm{N}$ antigens}

The primary objective of the initial mouse immunogenicity studies was to determine which of the rAd vectors induced significant antibody responses to $\mathrm{S}$, and to obtain those results rapidly enough to provide a GMP seed in time for manufacturing. We and others ${ }^{17}$ have observed that transgene expression by vaccine vectors orally administered to mice can be suppressed in their intestinal environment, so immunogenicity was assessed following intranasal (i.n.) immunization. Animals were immunized i.n. and the antibody titers were measured over time by IgG ELISA. All three rAd vectors induced nearly equivalent anti-S1 IgG titers, at weeks 2 and 4 and the $\operatorname{IgG}$ titer in all animals was significantly boosted by the second immunization $(\mathrm{p}<0.05$ Mann Whitney t-tests) (Fig. 2A). However, the vector expressing full-length $\mathrm{S}$ (rAd-S-N) induced higher neutralizing titers compared to the vector expressing only S1 (Fig 2B). This was measured by two different neutralizing assays, one based on SARS-CoV-2 infection of Vero cells (cVNT) and one based on a surrogate neutralizing assay (sVNT). Furthermore, rAd-S-N induced higher lung IgA responses to S1 and unsurprisingly, to S2 (Fig. 2C) compared to rAd-S1-N two weeks after the final immunization. Notably, neutralizing titers in the lung were also significantly higher when rAd-S-N was used compared to the S1-containing vaccine (rAd-S1-N) (Fig. 2D). This demonstrated that the rAd-S-N candidate induced greater functional responses (NAb and $\operatorname{IgA}$ ) 
compared to the vaccine containing the just the $\mathrm{S} 1$ domain. Because the $\mathrm{N}$ protein is much more highly conserved than the $\mathrm{S}$ protein, and is a target of long term $\mathrm{T}$ cell responses induced by infection ${ }^{18}$, the vector rAd-S-N was chosen for GMP manufacturing.

A



$\mathrm{C}$

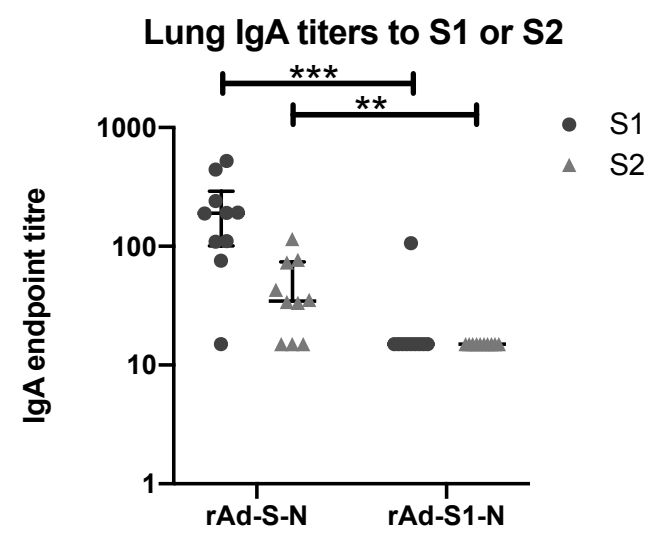

B

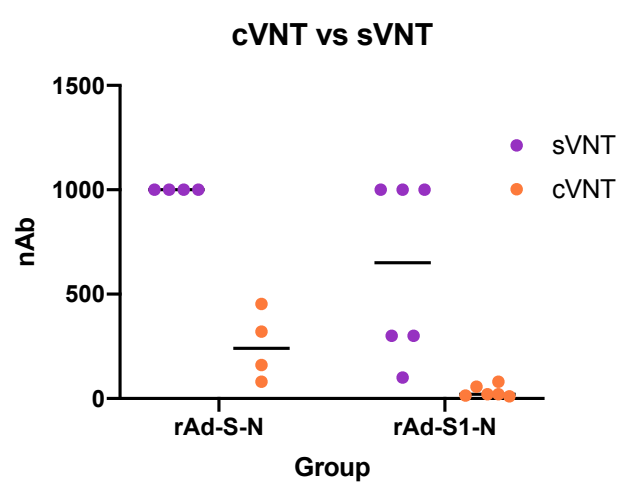

$\mathrm{D}$

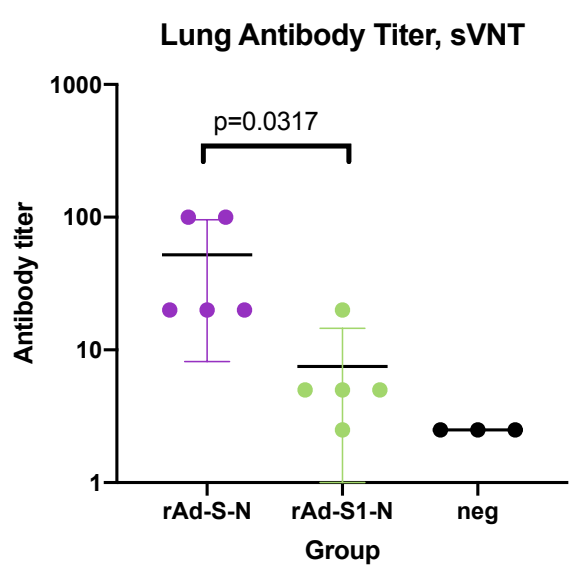

Figure 2. Immunization with candidate $r A d$ vaccines induce serum IgG and lung IgA responses. Antibody titers to $S$ following immunization of Balb/c mice on days 0 and 14 with $1 \times 10^{8}$ IU rAd expressing full-length $S$ (rAd-S), co-expressing full length $S$ and $N(r A d-S-N)$ or co-expressing a fusion protein comprising the $S 1$ domain and $N$ (rAd-S1-N). (A) IgG serum IgG endpoint titers to S1 were measured by standard ELISA ( $n=6$ per vaccinated group, $n=3$ for PBS administered group). Symbols represent mean titers and bars represent the standard error) (B) Neutralizing antibody responses comparing $r A d-S-N$ and $r A d-S 1-N$ using two different methods, surrogate VNT (sVNT) and cell-based VNT (cVNT). (C) IgA lung antibody titers to S1 and S2 in immunized mice. Endpoint titers were measured by standard ELISA ( $n=10$ per group). Lines represent the median and inter-quartile range. ** $p<0.01,{ }^{* * *} p<0.001$ defined by Mann-Whitney t-test. D. Neutralizing antibodies measured in the lungs post immunization. 
Three dose levels of rAd-S-N were then tested to understand the dose responsiveness of this vaccine. The antibody responses to both S1 (Fig. 3A) and S2 (Fig. 3B) were measured. Similar responses were seen at all three dose levels at all timepoints. Responses to S1 and S2 were significantly increased at week 6 compared to earlier times, in all groups.

The induction of S-specific T cells by rAd-S-N at different doses was then assessed. Induction of antigen-specific CD4+ and CD8 + T cells that produced effector cytokines such as IFN- $\gamma$, TNF- $\alpha$ and IL-2 was observed two weeks after 2 immunizations (Fig 4A). Notably, little IL-4 was induced by this vaccine and only in CD4+ T cells; providing a level of assurance that the risk for vaccine dependent enhancement of disease was very low. Furthermore, immunization with rAd$\mathrm{S}-\mathrm{N}$ induced double and triple positive, multi-functional IFN- $\gamma$, TNF- $\alpha$ and IL-2 CD4+ T cells (Fig. 4B). A second dose response experiment was performed to focus on $\mathrm{T}$ cell responses to the $\mathrm{S}$ protein, 4 weeks after the final immunization (week 8 of the study). Splenocytes were stimulated overnight with a peptide library to the $\mathrm{S}$ protein, divided in two separate peptide pools. $\mathrm{T}$ cell responses in the two pools were summed and plotted (Fig. 4C). Animals administered the $1 \mathrm{e} 7 \mathrm{IU}$ and the 1e8 IU dose levels had significantly higher $\mathrm{T}$ cell responses compared to the untreated animals but produced a similar number of IFN- $\gamma$ secreting cells to each other, demonstrating a dose plateau at the $1 \mathrm{e} 7 \mathrm{IU}$ dose. Notably, this $\mathrm{T}$ cell analysis was conducted 4 weeks after the second immunization, potentially after the peak of $\mathrm{T}$ cell responses.

A

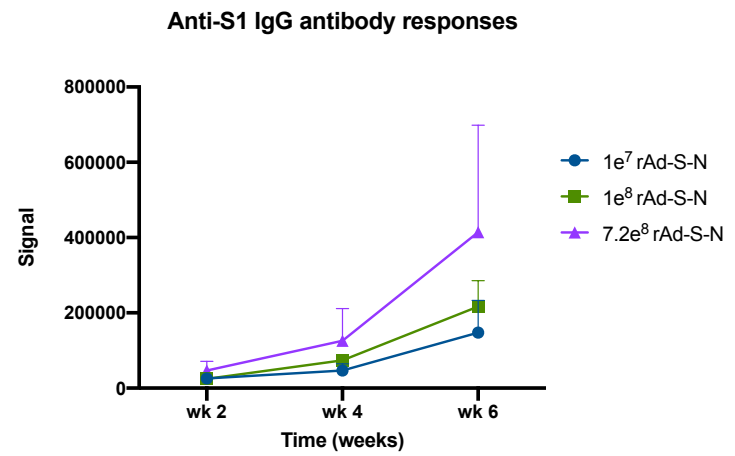

$\mathrm{B}$

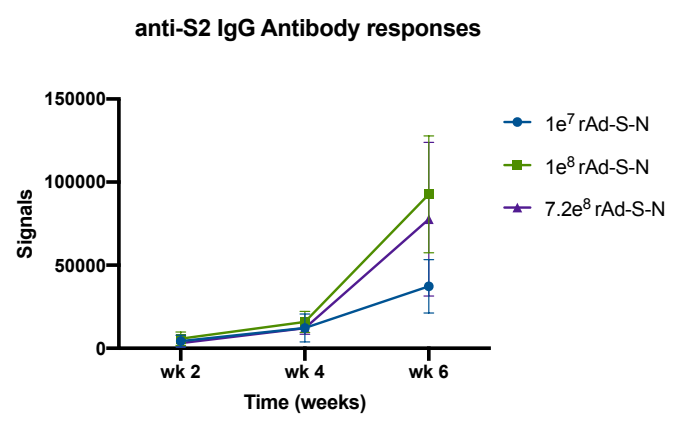

Figure 3. Immunization with rAd co-expressing full length $S$ and $N$ vaccines induce IgG responses in a dosedependent manner. A and B. Balb/c mice were immunized, IN, on days 0 and 14 with $1 \times 10^{7} \mathrm{IU}, 1 \times 10^{8} \mathrm{IU}$ or $7.2 \times$ $10^{8} \mathrm{IU}$ of $\mathrm{rAd}$ co-expressing full length $S$ and $N(\mathrm{rAd}-\mathrm{S}-\mathrm{N})$. The amount of IgG specific for $S 1(\boldsymbol{A})$ and $S 2$ in serum diluted 1/4000, was evaluated using a Mesoscale binding assay. Points represent the mean and lines represent the standard deviation. 

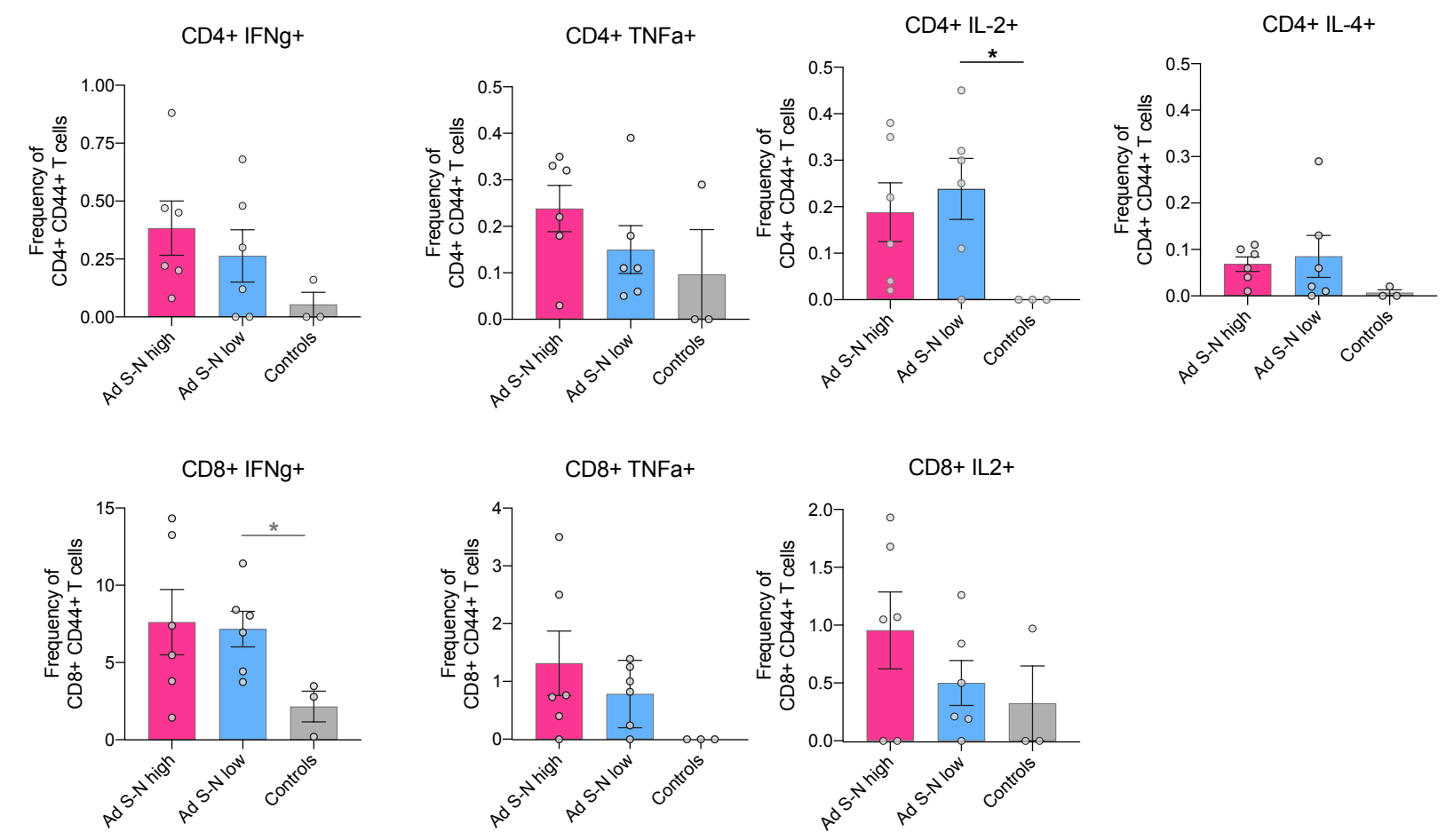

B.

$\mathrm{CD} 4+$ Triple+ $(\mathrm{IFNg}+\mathrm{IL} 2+\mathrm{TNFa}+)$


CD8+ Double+ (IFNg+ TNFa+)

$\mathrm{CD} 8+$ Triple+ $(\mathrm{IFNg}+\mathrm{TNFa}+\mathrm{IL}-2+)$
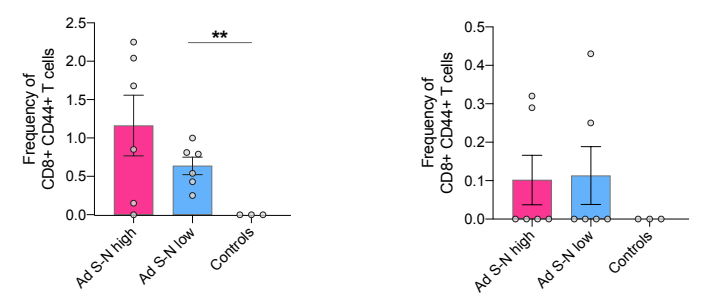

C.

IFN-g T cell Responses to S

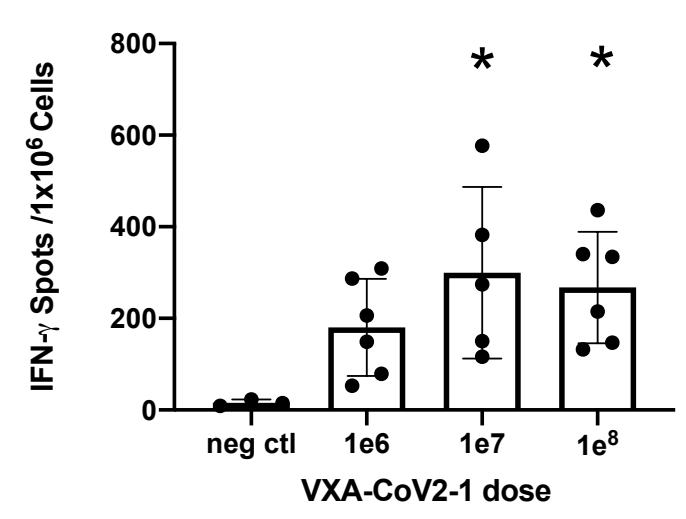

Figure 4. Immunization with $r A d$ co-expressing full length $S$ and $N$ vaccines induce polyfunctional $T$ cell responses. (A) Balb/c mice were immunized, IN, on days 0 and 14 with $1 \times 10^{8} \mathrm{IU}$ (Ad-S-N high), $1 \times 10^{7} \mathrm{IU}$ (Ad-S-N low) of rAd$S-N$. The frequency of CD4+ (top panel) or CD8+ T cells (bottom panel) that produced only IFN- $\gamma, T N F-\alpha, I L-2$ or IL4 after stimulation of spleen cells with $1 \mu \mathrm{g} / \mathrm{ml}\left(C D 4^{+}\right)$or $5 \mu \mathrm{g} / \mathrm{ml}\left(C D 8^{+}\right)$of the $S$ peptide pools, as determined by ICSFACS. (B) The frequency of polyfunctional CD4+ (top panel) or CD8+ T cells (bottom panel) that produced more than 
one cytokine after stimulation of spleen cells, Bars represent the mean and the lines represent the standard error of the mean. (C) IFN- $\gamma$ T cell responses to S protein 4 weeks following immunization on weeks 0 and 4 with $1 \times 10^{6} \mathrm{IU}, 1 \times$ $10^{7} \mathrm{IU}, 1 \times 10^{8} \mathrm{IU}$ doses of $\mathrm{rAd}-\mathrm{S}-\mathrm{N}$ were measured by ELISPOT. Bars represent the mean and the lines represent the standard deviation. * $p<0.05$; one-way non-parametric ANOVA with multiple comparisons.

\section{rAd-expressed wild-type $S$ induces a superior neutralizing response compared to stabilized/pre-fusion $S$.}

An additional study was performed to compare rAd-S-N to a vaccine candidate with the S-protein stabilized and with the transmembrane region removed (rAd-S(fixed)-N). A stabilized version of the $\mathrm{S}$ protein has been proposed as a way to improve neutralizing antibody responses and produce less non-neutralizing antibodies. The $\mathrm{S}$ protein was stabilized through modifications as described by Amanat et al., ${ }^{19}$. rAd-S-N induced higher serum IgG titers to S1 (Fig. 5A) at both timepoints tested, although these were not statistically significant at week 6 by Mann-Whitney $(\mathrm{p}=0.067)$. However, rAd-S-N induced significantly higher neutralizing antibody responses (Fig. 5B) than the stabilized version $(\mathrm{p}=0.0152)$. These results suggest that a wild-type version of the $\mathrm{S}$ protein is superior for a rAd based vaccine in mice.

A

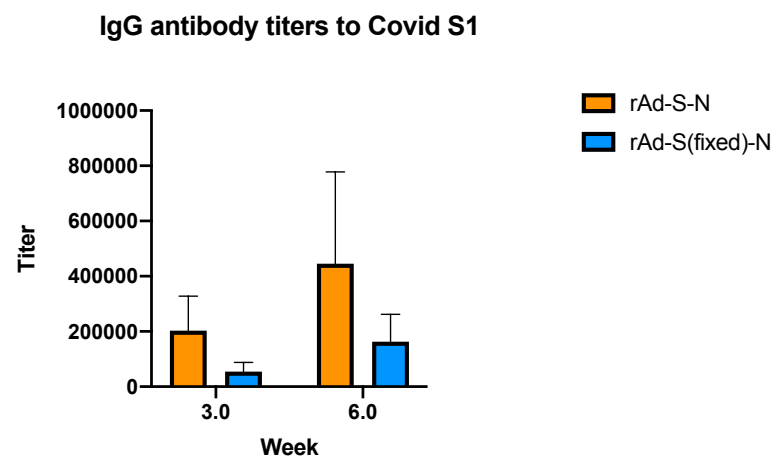

B

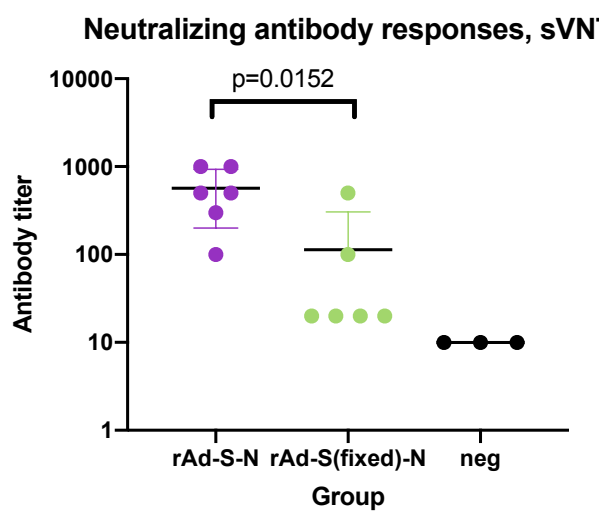

Figure 5: Antibodies to $S$ were superior when the $S$ protein expressed in the wild-type configuration compared to the fixed version. Balb/c mice were immunized on weeks 0 and 4 with le8 IU per mouse ( $n$ $=6$ ), and antibody titers were measured. (A) IgG antibody titers over time. (B) Neutralizing antibody responses were measured at week 6 . Note that 1:1000 was the maximum dilution performed.

\section{DISCUSSION}

The endgame to the COVID-19 pandemic requires the identification and manufacture of a safe and effective vaccine and a subsequent global immunization campaign. A number of vaccine candidates have accelerated to phase III global efficacy testing and, if sufficiently successful in these trials, may form the first generation of an immunization campaign. However, all of these advanced candidates are S-based vaccines that are injected. Such an approach will unlikely prevent virus transmission, but 
should prevent pneumonia and virus growth and damage in the lower respiratory tract and periphery, as evidenced in macaque challenge studies ${ }^{4,5}$.

One key constraint in a global COVID-19 immunization campaign will be the cold chain distribution logistics and a bottleneck of requiring suitably trained health care workers (HCWs) to inject the vaccine. Current logistics costs, including cold chain and training, can double the cost of fully immunizing an individual in a low-middle income country (LMIC) ${ }^{20}$. Implementing a mass immunization campaign, requiring trained $\mathrm{HCW}$ for injection-based vaccines, will have a significant impact on healthcare resources in all countries. The need for cold chain, biohazardous sharps waste disposal and training will result in increased cost, inequitable vaccine access, delayed vaccine uptake and prolongation of this pandemic. These costs will be magnified if vaccines are unable to provide long-term protection (natural immunity to other beta-coronaviruses is short-lived ${ }^{21}$ ), and annual injection-based campaigns are needed. Vaxart's oral tablet vaccine platform provides a solution to these immunological as well as logistic, economic, access and acceptability problems. In this study we demonstrate, in an animal pre-clinical model, the immunogenicity of a SARS-CoV-2 vaccine using Vaxart's vaccine platform; namely the induction of serum and mucosal neutralising antibodies and poly-functional $\mathrm{T}$ cells.

Mouse studies were designed to test immunogenicity of candidate vaccines rapidly in the spring of 2020, before moving onto manufacturing and clinical studies critical to addressing the pandemic. Vaxart's oral tablet vaccine platform has previously proven to be able to create reliable mucosal (respiratory and intestinal), $\mathrm{T}$ cell, and antibody responses against several different pathogens in humans ${ }^{12,14,22,23}$. We know from our prior human influenza virus challenge study that oral immunization was able to induce protective efficacy 90 days post immunization; on par with the commercial quadrivalent inactivated vaccine ${ }^{15}$. These features provide confidence that the adoption of the platform to COVID-19 could translate to efficacy against this pathogenic coronavirus and could provide durable protection against virus infection. Finally, a tablet vaccine campaign is much easier because qualified medical support is not needed to administer it. This ease of administration will result in increased vaccine access and potentially, acceptability, as has been evidenced by the success of easy-to-administer, oral polio vaccine, in the elimination of polio virus ${ }^{24}$. These features could be even more important during SARS-CoV-2 immunization campaigns compared to other vaccines, as substantially more resources may be required to ensure uptake of this vaccine, given the global levels of COVID-19 denialism, mistrust and increased vaccine hesitancy ${ }^{25,26}$. The tablet vaccine does not need refrigerators or freezers, does not require needles or vials, and can potentially be shipped via standard mail or by a delivery drone. These attributes significantly enhance deployment and distribution logistics, even permitting access to isolated regions with fewer technical resources. Finally, from an immunological perspective, oral administration of this adenovirus is not compromised by pre-existing immunity to adenoviruses nor creates substantial anti-vector immunity $^{12,13}$; issues that have been shown to cause significantly decreased vaccine potency in an rAd5 based SARS-CoV2 vaccine ${ }^{27}$ and can prevent durable increased immunity when the same adenovirus platform is re-administered by the IM route ${ }^{28}$. 
The choice of antigen can be difficult during a novel pandemic, a time in which key decisions are needed quickly. The $\mathrm{S}$ protein is believed to be the major neutralizing antibody target for coronavirus vaccines, as the protein is responsible for receptor binding, membrane fusion, and tissue tropism. When comparing SARS-CoV-2 Wu-1 to SARS-CoV, the $\mathrm{S}$ protein was found to have $76.2 \%$ identity $^{29}$. Both SARS-CoV and SARS-CoV-2 are believed to use the same receptor for cell entry: the angiotensin-converting enzyme 2 receptor (ACE2), which is expressed on some human cell types ${ }^{30}$. Thus, SARS-CoV-2 S protein is being used as the leading target antigen in vaccine development so far and is an ideal target given that it functions as the key mechanism for viral binding to target cells. However, the overall reliance on the $\mathrm{S}$ protein and an $\mathrm{IgG}$ serum response in the vaccines could eventually lead to viral escape. For influenza, small changes in the hemagglutinin binding protein, including a single glycosylation site, can greatly affect the ability of injected vaccines to protect ${ }^{31}$. SARS-CoV-2 appears to be more stable than most RNA viruses, but $\mathrm{S}$ protein mutations have already been observed without the selective pressure of a widely distributed vaccine. Once vaccine pressure begins, escape mutations might emerge. We took two approaches to address this issue; firstly to include the more conserved $\mathrm{N}$ protein in the vaccine and secondly to induce broader immune responses, namely through mucosal $\operatorname{IgA}$.

High expression levels of ACE2 are present in type II alveolar cells of the lungs, absorptive enterocytes of the ileum and colon, and possibly even in oral tissues such as the tongue ${ }^{32}$. Transmission of the virus is believed to occur primarily through respiratory droplets and fomites between unprotected individuals in close contact ${ }^{33}$, although there is some evidence of transmission via the oral-fecal route as seen with both SARS-CoV and MERS-CoV viruses where coronaviruses can be secreted in fecal samples from infected humans ${ }^{34}$. There is also evidence that a subset of individuals exist that have gastrointestinal symptoms, rather than respiratory symptoms, are more likely to shed virus longer ${ }^{35}$. Driving immune mucosal immune responses to $\mathrm{S}$ at both the respiratory and the intestinal tract may be able provide broader immunity and a greater ability to block transmission, than simply targeting one mucosal site alone. Blocking transmission, rather than just disease, will be essential to reducing infection rates and eventually eradicating SARS-CoV-2. We have previously demonstrated that an oral, tableted rAd-based vaccine can induce protection against respiratory infection and shedding following influenza virus challenge ${ }^{15}$ as well as intestinal immunity to norovirus antigens in humans ${ }^{12}$. Furthermore, mucosal IgA is more likely to be able address any heterogeneity of the $\mathrm{S}$ proteins in circulating viruses than a monomeric IgG response. $\mathrm{mIgA}$ has also been found to be more potent at cross reactivity than IgG for other respiratory pathogens $^{36}$. IgA may also be a more neutralizing isotype than IgG in COVID-19 infection, and in fact neutralizing IgA dominates the early immune response ${ }^{37}$. Notably, we saw a higher ratio between neutralizing to non-neutralizing antibodies in our lung versus serum antibody results in our mouse study as well, which supports the concept that IgA may have more potency compared to IgG. Polymeric IgA, through multiple binding interactions to the antigen and to Fc receptors can turn a weak single interaction into a higher overall affinity binding and activation signal, creating more cross-protection against heterologous viruses ${ }^{38}$. 
Our second strategy to mitigate this potential vaccine-driven escape problem was to include the $\mathrm{N}$ protein in the vaccine construct. The $\mathrm{N}$ protein is highly conserved among $\beta$-coronaviruses, (greater than $90 \%$ identical) contains several immunodominant $\mathrm{T}$ cell epitopes, and long-term memory to $\mathrm{N}$ can be found in SARS-CoV recovered subjects as well as people with no known exposure to SARS$\mathrm{CoV}$ or SARS-CoV-2 ${ }^{18,39}$. In an infection setting, $\mathrm{T}$ cell responses to the $\mathrm{N}$ protein seem to correlate to increased neutralizing antibody responses ${ }^{40}$. All of these reasons led us to add $\mathrm{N}$ to our vaccine approach. The protein was expressed in 293A cells (Supp Fig1). However, as the human beta actin promoter is more active in human cells than mice, we did not explore immune responses in Balb/c mice, but will examine them more carefully in future NHP and human studies.

The optimum sequence and structure of the $\mathrm{S}$ protein to be included in a SARS-CoV-2 vaccine is a subject of debate. Several labs have suggested that reducing the S protein to the key neutralizing domains within the receptor binding domain (RBD) would promote higher neutralizing antibody responses, and fewer non-neutralizing antibodies ${ }^{41,42}$. We made a vaccine candidate composed of the $\mathrm{S} 1$ domain, which includes the RBD, in an attempt to promote this approach. Although the S1based vaccine produced similar IgG binding titers to $\mathrm{S} 1$, neutralizing antibody responses were significantly lower compared to the full-length $\mathrm{S}$ antigen. Other gene-based vaccines have also shown the reductionist approach to $\mathrm{S}$ does not work so well, demonstrating that the DNA vaccine expressing the full-length S-protein produced higher neutralizing antibodies than shorter S segments ${ }^{5}$. In agreement with these macaque studies, we observed that the sequence of the adenovirus encoded antigen had a significant impact on antibody function, here with respect to neutralization. While reducing the potential for exposing non-neutralizing antibody epitopes seems reasonable in theory, this might reduce the $\mathrm{T}$ cell help that allows for greater neutralizing antibodies to develop. Indeed, of the spike protein $\mathrm{T}$ cell responses, which make up 54\% of the responses to SARS-CoV-2, only $11 \%$ map to receptor binding domain ${ }^{43}$. Stabilizing the $\mathrm{S}$ protein might be important for a protein vaccine, but not necessarily for a gene-based vaccine. The former is produced in vitro and it is produced to retain a homogenous, defined structure, ready for injection. In contrast, the latter, is expressed on the surface of a cell, in vivo, like natural infection, substantially in a prefusion form, and the additional stabilization may be unnecessary for B cells to create antibodies against the key neutralizing epitopes. We directly compared a stabilized version of $\mathrm{S}$ to the wild-type version. The wild-type version was significantly better at inducing neutralizing antibody responses. Of interest, this was also observed in a DNA vaccine study in NHPs, where the stabilized version appeared to induce lower neutralizing antibody $(\mathrm{NAb})$ titers compared to the wild-type $\mathrm{S}^{5}$. A slightly different result was observed in studies of rAd26 vectors by Mercado, et al in NHPs, where expressing a stabilized version of the $\mathrm{S}$ protein appeared to improve NAb but lower $\mathrm{T}$ cell responses ${ }^{44}$. In summary, stabilization doesn't universally improve the immune responses in gene-based or vectorbased vaccines.

Multiple vaccine candidates are in, or are about to begin, clinical testing. Due to known safety and immunogenicity for epidemic pathogens such as Ebola virus, two leading candidate vaccines are based on recombinant adenovirus vectors; University of Oxford's ChAdOx1-nCov and Janssen 
Pharmaceutical's AdVac platforms ${ }^{45-48}$. We saw stronger serum IgG and NAb titers in our study compared to a ChAdOx1-nCov in Balb/c mice ${ }^{4}$, however, this might reflect differences in assay components. A rAd36 vaccine study was performed by Hassan, et al., where doses of 1e10 VP were given by intranasal delivery ${ }^{49}$. The results were significant from the standpoint of blocking lung infection in a mouse SARS-CoV-2 challenge model. They reported titers of serum antibody titers of 1e4 above the background titers, similar to our results, despite using doses 2- to 3-log fold higher viral doses compared to our study. Indeed, in our study, equivalently strong $\mathrm{T}$ cell and antibody responses were observed using $1 \mathrm{e} 7 \mathrm{IU}$ and $1 \mathrm{e} 8 \mathrm{IU}$ by the intranasal route. Using these doses, we observed high percentages of CD8+ T cell responses (up to 14\%) secreting IFN- $\gamma$ and TNF- $\alpha$ and strong CD4+ $\mathrm{T}$ cells after peptide restimulation. Although we did not evaluate the trafficking properties of these antigen-specific T cells, we know that oral administration of this Ad-based vaccine in humans induces high levels of mucosal homing lymphocytes ${ }^{12,15}$. A proportion of the antigenspecific CD4+ and CD8+ T cells were polyfunctional in this mouse study. Vaccine-induced T cells possessing multiple functions may provide more effective elimination of virus subsequent to infection and therefore could be involved in the prevention of disease. However, it is uncertain at this time what is the optimum $\mathrm{T}$ cell phenotype required for protection against disease.

In summary, these studies in mice represent our first step in creating a vaccine candidate, demonstrating the immunogenicity of the construct at even low vaccine doses and the elucidation of the full-length spike protein as a leading candidate antigen to induce $\mathrm{T}$ cell responses and superior systemic and mucosal neutralizing antibody. Future work will focus on the immune responses in humans.

\section{METHODS}

\section{Vaccine Constructs}

For this study, four recombinant adenoviral vaccine constructs were created based on the published DNA sequence of SARS-CoV-2 publicly available as Genbank Accession No. MN908947.3. Specifically, the published amino acid sequences of the SARS-CoV-2 spike protein (S protein) and the SARS-CoV-2 Nucleocapsid protein (N protein) were used to synthesize nucleic acid sequences codon optimized for expression in Homo sapiens cells (Blue Heron Biotechnology, Bothell, WA). These sequences were used to create recombinant plasmids containing transgenes cloned into the E1 region of Adenovirus Type 5 (rAd5), as described by He, et al ${ }^{50}$, using the same vector backbone used in prior clinical trials for oral rAd tablets ${ }^{12,15}$. As shown in Fig 1, the following four constructs were created:

a. rAd-S: rAd5 vector containing full-length SARS-CoV-2 S gene under control of the CMV promoter.

b. rAd-S-N: rAd5 vector containing full-length SARS-CoV-2 S gene under control of the CMV promoter and full-length SARS-CoV-2 N gene under control of the human betaactin promoter. 
c. rAd-S1-N: rAd5 vector using a fusion sequence combining the S1 region of SARS-CoV$2 \mathrm{~S}$ gene (including the native furin site between S1 and S2) with the full-length SARSCoV-2 N gene.

d. rAd-S(fixed)-N: rAd5 vector containing a stabilized $\mathrm{S}$ gene with the transmembrane region removed under the control of the CMV promoter and full-length SARS-CoV-2 N gene under control of the human beta-actin promoter. The $\mathrm{S}$ gene is stabilized through the following modifications:

a) Arginine residues at aa positions $682,683,685$ were deleted to remove the native furin cleavage site

b) Two stabilizing mutations were introduced: K986P and V987P

c) Transmembrane region was removed following P1213 and replaced with bacteriophage T4 fibritin trimerization foldon domain sequence ${ }^{51}$ (GYIPEAPRDGQAYVRKDGEWVLLSTFL)

All vaccines were grown in the Expi293F suspension cell-line (Thermo Fisher Scientific), purified by $\mathrm{CsCl}$ density centrifugation and provided in a liquid form for animal experiments.

\section{Animal Experiments}

Studies were approved for ethics by the Animal Care and Use Committees (IACUC). All of the procedures were carried out in accordance with local, state and federal guidelines and regulations. Female 6-8 week old Balb/c mice were purchased from Jackson labs (Bar Harbor, $\mathrm{ME})$. Because mice do not swallow pills, liquid formulations were instilled intranasally in 10 $\mu l$ per nostril, $20 \mu \mathrm{l}$ per mouse in order to test immunogenicity of the various constructs. Serum was acquired by cheek puncture at various timepoints.

\section{Antibody Assessment}

ELISAS.

Specific antibody titers to proteins were measured similarly to methods described previously ${ }^{52}$. Briefly, microtiter plates (MaxiSorp: Nunc) were coated in 1 carbonate buffer (0.1 $\mathrm{M}$ at $\mathrm{pH} 9.6)$ with $1.0 \mathrm{ug} / \mathrm{ml} \mathrm{S} 1$ protein (GenScript). The plates were incubated overnight at $4{ }^{\circ} \mathrm{C}$ in a humidified chamber and then blocked in PBS plus $0.05 \%$ Tween 20 (PBST) plus 1\% BSA solution for $1 \mathrm{~h}$ before washing. Plasma samples were serially diluted in PBST. After a 2-h incubation, the plates were washed with PBST at least 5 times. Antibodies were then added as a mixture of anti-mouse IgG1-horseradish peroxidase (HRP) and anti-mouse IgG2a-HRP (Bethyl Laboratories, Montgomery, TX). Each secondary antibody was used at a 1:5,000 dilution. The plates were washed at least 5 times after a 1-h incubation. Antigen-specific mouse antibodies were detected with 3,3=,5,5=-tetramethylbenzidine (TMB) substrate (Rockland, Gilbertsville, PA) and $\mathrm{H}_{2} \mathrm{SO}_{4}$ was used as a stop 
solution. The plates were read at $450 \mathrm{~nm}$ on a Spectra Max M2 Microplate Reader. Average antibody titers were reported as the reciprocal dilution giving an absorbance value greater than the average background plus 2 standard deviations, unless otherwise stated.

\section{Antibody binding antibodies}

To measure responses to both S1 and S2 simultaneously, A MULTI-SPOT® 96-well, 2-Spot Plate (Mesoscale Devices; MSD) was coated with SARS CoV-2 antigens. Proteins were commercially acquired from a source (Native Antigen Company) that produced them in mammalian cells (293 cells). These were biotinylated and adhered to their respective spots by their individual U-PLEX linkers. To measure IgG antibodies, plates were blocked with MSD Blocker B for 1 hour with shaking, then washed three times prior to the addition of samples, diluted 1:4000. After incubation for 2 hours with shaking, the plates were washed three times. The plates were then incubated for 1 hour with the detection antibody at $1 \mu \mathrm{g} / \mathrm{mL}$ (MSD SULFO-TAG ${ }^{\mathrm{TM}}$ Anti-mouse IgG). After washing 3 times, the Read Buffer was added and the plates were read on the Meso QuickPlex SQ 120.

\section{SARS-CoV-2 Neutralization Assays}

Neutralizing antibodies were routinely detected based on the SARS-CoV-2 Surrogate Virus Neutralization Test (sVNT) kit (GenScript). This ELISA-based kit detects antibodies that hinder the interaction between the receptor binding domain (RBD) of the SARS-CoV-2 spike glycoprotein and the ACE2 receptor on host cells, and is highly correlated to conventional virus neutralizing titers for SARS-CoV-2 infection of Vero cells ${ }^{53}$. The advantage of this approach is that the assay can be done in a BSL-2 laboratory. Sera from mice immunized with the candidate vaccines was diluted at 1:20, 1:100, 1:300, 1:500, 1:750 and 1:1000 using the provided sample dilution buffer. Sera from non-immunized mice was diluted 1:20. Lung samples were diluted $1: 5,1: 20$, and 1:100. Positive and negative controls were prepared at a 1:9 volume ratio following the provided protocol. After dilution, sera or lung samples were individually incubated at a $1: 1$ ratio with $\mathrm{HRP}-\mathrm{RBD}$ solution for 30 minutes at $37^{\circ} \mathrm{C}$. Following incubation, $100 \mu l$ of the each HRP-RBD and sample or control mixture was added to the corresponding wells in the hACE2-precoated capture plate and once again incubated at $37^{\circ} \mathrm{C}$ for 15 minutes. Afterwards, wells were thoroughly washed and $100 \mu \mathrm{l}$ of the provided $\operatorname{TMB}(3,3=, 5,5=-$ tetramethyl-benzidine) solution was added to each well and left to incubate for 15 minutes at room temperature $\left(20-25^{\circ} \mathrm{C}\right)$. Lastly $50 \mu 1$ of Stop Solution was added to each well, and the plate was read on a Spectra Max M2 Microplate Reader at $450 \mathrm{~nm}$. The absorbance of a given sample is inversely related on the titer of anti- SARS-CoV-2 RBD neutralizing antibody in a given sample. Per test kit protocol, a cut-off of $20 \%$ inhibition when comparing the OD of the sample versus the $\mathrm{OD}$ of the negative control was determined to be positive for the presence of 
neutralizing antibodies. Samples that were negative at the lowest dilution were set equal to $1 / 2$ of the lowest dilution tested, either 10 for sera or 2.5 for lung samples.

Additional neutralizing antibodies responses were measured in some studies using a cVNT assay at Visimederi under BSL3 conditions. The cVNT assay has a readout of Cytopathic Effect (CPE) to detect specific neutralizing antibodies against live SARS-COV-2 in animal or human samples. The cVNT/CPE assay permits the virus to makes multiples cycles of infection and release from cells; its exponential grow in few days (usually 72 hours of incubation) causes the partial or complete cell monolayer detachment from the surface of the support, clearly identifiable as CPE. Serum samples are heat inactivated for $30 \mathrm{~min}$ at $56^{\circ}$; two-fold dilutions, starting from 1:10 are performed then mixed with an equal volume of viral solution containing $100 \mathrm{TCID}_{50}$ of SARS-CoV-2. The serum-virus mixture is incubated for 1 hour at $37^{\circ}$ in humidified atmosphere with $5 \% \mathrm{CO} 2$. After incubation, $100 \mu \mathrm{L}$ of the mixture at each dilution are added in duplicate to a cell plate containing a semiconfluent Vero E6 monolayer. After 72 hours of incubation the plates are inspected by an inverted optical microscope. The highest serum dilution that protect more than $50 \%$ of cells from CPE is taken as the neutralization titer.

\section{Lung IgA ELISAs.}

Two weeks after the final immunization (day 28 of the study), mice were sacrificed and bled via cardiac puncture. Lungs were removed and snap frozen at $-80{ }^{\circ} \mathrm{C}$. On thawing, lungs were weighed. Lungs were homogenized in $150 \mu$ DPBS using pellet pestles (Sigma Z359947). Homogenates were centrifuged at 1300rpm for 3 minutes and supernatants were frozen. The total protein content in lung homogenate was evaluated using a Bradford assay to ensure equivalent amounts of tissue in all samples before evaluation of IgA content. Antigen-specific IgA titers in lungs were detected using a mouse IgA ELISA kit (Mabtech) and pNPP substrate (Mabtech). Briefly, MaxiSorp plates (Nunc) were coated with S1 or S2 (The Native Antigen Company; 50 ng/well) in PBS for overnight adsorption at $4^{\circ} \mathrm{C}$ and then blocked in PBS plus $0.05 \%$ Tween 20 (PBST) plus $0.1 \% \mathrm{BSA}(\mathrm{PBS} / \mathrm{T} / \mathrm{B})$ solution for $1 \mathrm{~h}$ before washing. Lung homogenates were serially diluted in $\mathrm{PBS} / \mathrm{T} / \mathrm{B}$, starting at a 1:30 dilution. After 2 hours incubation and washing, bound IgA was detected using MT39A-ALP conjugated antibody (1:1000), according to the manufacturer's protocol. Plates were read at $415 \mathrm{~nm}$. Endpoint titers were taken as the $\mathrm{x}$-axis intercept of the dilution curve at an absorbance value 3x standard deviations greater than the absorbance for naïve mouse serum. Non-responding animals were set a titer of 15 or $1 / 2$ the value of the lowest dilution tested.

\section{T cell Responses}

Spleens were removed and placed in $5 \mathrm{ml}$ Hanks Balanced Salt Solution (with 1M HEPES and 5\% FBS) before pushing through a sterile strainer with a $5 \mathrm{ml}$ syringe. After RBC lysis (Ebiosolutions), resuspension, and counting, the cells were ready for analysis. Cells were 
cultured at 5e5 cells/well with two peptide pools representing the full-length S protein at 1 $\mu \mathrm{g} / \mathrm{ml}$ (Genscript) overnight in order to stimulate the cells. The culture media consisted of RPMI media (Lonza) with 0.01M HEPES, 1X 1-glutamine, 1X MEM basic amino acids, 1X penstrep, 10\% FBS, and 5.5e-5 mole/1 beta-mercaptoethanol. Antigen specific IFN- $\gamma$ ELISPOTs were measured using a Mabtech kit. Flow cytometric analysis was performed using an Attune Flow cytometer and Flow Jo version 10.7.1, after staining with the appropriate antibodies. For flow cytometry, 2e6 splenocytes per well were incubated for 18 hours at $37^{\circ} \mathrm{C}$ with peptide pools representing full-length $\mathrm{S}$ at either 1 or $5 \mathrm{ug} / \mathrm{ml}$, adding Brefeldin A (ThermoFisher) for the last 4 hours of incubation. The antibodies used were APC-H7 conjugated CD4, FITC conjugated CD8, BV650 conjugated CD3, PerCP-Cy5.5 conjugated IFN-y, BV421 conjugated IL-2, PE-Cy7 conjugated TNFa, APC conjugated IL-4, Alexa Fluor conjugated CD44, and PE conjugated CD62L (BD biosciences).

\section{ACKNOWLEDGEMENTS}

The authors would like to thank Alessandro Torelli, Laura Palladino, Emanuele Montomoli and the team at VisMederi for running neutralizing antibody titers (BSL3) and Susan Johnson for critically reviewing the manuscript.

\section{AUTHORSHIP}

ACM, MC, and SNT designed the experiments; ACM and SNT wrote the manuscript; NP, KPT, and KL performed immunological assays; ACM, NP, KPT, KL, MC, and SNT analyzed the data. EGD and SNT designed the vaccine vectors.

\section{COMPETING INTERESTS}

EGD, ND, KPT, KLM MC and SNT are current employees and/or own stock options in Vaxart, the sponsor of the studies. EGD and SNT are named as inventors covering a $S A R S$ $\mathrm{CoV}-2(n \mathrm{CoV}-19)$ vaccine. SNT is named as an inventor on patent covering the vaccine platform. ACM declares no competing interest.

\section{REFERENCES}

1. Huang C, Wang Y, Li X, et al. Clinical features of patients infected with 2019 novel coronavirus in Wuhan, China. Lancet 2020; 395(10223): 497-506.

2. Tan J, Liu S, Zhuang L, et al. Transmission and clinical characteristics of asymptomatic patients with SARS-CoV-2 infection. Future Virol 2020: 10.2217/fvl-020-0087.

3. Long QX, Tang XJ, Shi QL, et al. Clinical and immunological assessment of asymptomatic SARS-CoV-2 infections. Nat Med 2020; 26(8): 1200-4. 
4. van Doremalen N, Lambe T, Spencer A, et al. ChAdOx1 nCoV-19 vaccine prevents SARS-CoV-2 pneumonia in rhesus macaques. Nature 2020.

5. Yu J, Tostanoski LH, Peter L, et al. DNA vaccine protection against SARS-CoV-2 in rhesus macaques. Science 2020.

6. Folegatti PM, Ewer KJ, Aley PK, et al. Safety and immunogenicity of the ChAdOx1 nCoV-19 vaccine against SARS-CoV-2: a preliminary report of a phase $1 / 2$, single-blind, randomised controlled trial. Lancet 2020.

7. Zhu FC, Guan XH, Li YH, et al. Immunogenicity and safety of a recombinant adenovirus type-5-vectored COVID-19 vaccine in healthy adults aged 18 years or older: a randomised, double-blind, placebo-controlled, phase 2 trial. Lancet 2020; 396(10249): 479-88.

8. Jackson LA, Anderson EJ, Rouphael NG, et al. An mRNA Vaccine against SARS-CoV-2 - Preliminary Report. N Engl J Med 2020.

9. Renegar KB, Jackson GD, Mestecky J. In vitro comparison of the biologic activities of monoclonal monomeric IgA, polymeric IgA, and secretory IgA. Journal of immunology 1998; 160(3): 1219-23.

10. Seibert CW, Rahmat S, Krause JC, et al. Recombinant IgA is sufficient to prevent influenza virus transmission in guinea pigs. Journal of virology 2013; 87(14): 7793-804.

11. Lowen AC, Steel J, Mubareka S, Carnero E, Garcia-Sastre A, Palese P. Blocking interhost transmission of influenza virus by vaccination in the guinea pig model. J Virol 2009; 83(7): 2803-18.

12. Kim L, Liebowitz D, Lin K, et al. Safety and immunogenicity of an oral tablet norovirus vaccine, a phase I randomized, placebo-controlled trial. JCI Insight 2018; 3(13): e121077.

13. Liebowitz D, Lindbloom JD, Brandl JR, Garg SJ, Tucker SN. High titre neutralising antibodies to influenza after oral tablet immunisation: a phase 1, randomised, placebo-controlled trial. The Lancet infectious diseases 2015; 15(9): 1041-8.

14. Peters W, Brandl JR, Lindbloom JD, et al. Oral administration of an adenovirus vector encoding both an avian influenza A hemagglutinin and a TLR3 ligand induces antigen specific granzyme B and IFN-gamma T cell responses in humans. Vaccine 2013; 31: 1752-8.

15. Liebowitz D, Gottlieb K, Kolhatkar NS, et al. Efficacy and immune correlates of protection induced by an oral influenza vaccine evaluated in a phase 2 , placebo-controlled human experimental infection study. The Lancet infectious diseases 2020; 20: 435-44.

16. Yong CY, Ong HK, Yeap SK, Ho KL, Tan WS. Recent Advances in the Vaccine Development Against Middle East Respiratory Syndrome-Coronavirus. Front Microbiol 2019; 10: 1781 .

17. Revaud J, Unterfinger Y, Rol N, et al. Firewalls Prevent Systemic Dissemination of Vectors Derived from Human Adenovirus Type 5 and Suppress Production of TransgeneEncoded Antigen in a Murine Model of Oral Vaccination. Front Cell Infect Microbiol 2018; 8: 6. 18. Peng H, Yang LT, Wang LY, et al. Long-lived memory T lymphocyte responses against SARS coronavirus nucleocapsid protein in SARS-recovered patients. Virology 2006; 351(2): 466-75.

19. Amanat F, Stadlbauer D, Strohmeier S, et al. A serological assay to detect SARS-CoV-2 seroconversion in humans. medRxiv 2020. 
20. Gandhi G, Lydon P, Cornejo S, Brenzel L, Wrobel S, Chang H. Projections of costs, financing, and additional resource requirements for low- and lower middle-income country immunization programs over the decade, 2011-2020. Vaccine 2013; 31 Suppl 2: B137-48.

21. Kissler SM, Tedijanto C, Goldstein E, Grad YH, Lipsitch M. Projecting the transmission dynamics of SARS-CoV-2 through the postpandemic period. Science 2020; 368(6493): 860-8.

22. Kim L, Martinez CJ, Hodgson KA, et al. Systemic and mucosal immune responses following oral adenoviral delivery of influenza vaccine to the human intestine by radio controlled capsule. Scientific Reports 2016; 6(1): 37295.

23. Liebowitz D, Lindbloom JD, Brandl JR, Garg SJ, Tucker SN. High Titer Neutralizing Antibodies to Influenza Following Oral Tablet Immunization: A Randomized, Placebocontrolled Trial The Lancet infectious diseases 2015; 15: 1041-8.

24. Hird TR, Grassly NC. Systematic review of mucosal immunity induced by oral and inactivated poliovirus vaccines against virus shedding following oral poliovirus challenge. PLoS pathogens 2012; 8(4): e1002599.

25. Jaiswal J, LoSchiavo C, Perlman DC. Disinformation, Misinformation and InequalityDriven Mistrust in the Time of COVID-19: Lessons Unlearned from AIDS Denialism. AIDS Behav 2020.

26. Palamenghi L, Barello S, Boccia S, Graffigna G. Mistrust in biomedical research and vaccine hesitancy: the forefront challenge in the battle against COVID-19 in Italy. Eur J Epidemiol 2020.

27. Zhu FC, Li YH, Guan XH, et al. Safety, tolerability, and immunogenicity of a recombinant adenovirus type-5 vectored COVID-19 vaccine: a dose-escalation, open-label, nonrandomised, first-in-human trial. Lancet 2020; 395(10240): 1845-54.

28. Baden LR, Walsh SR, Seaman MS, et al. First-in-Human Evaluation of the Safety and Immunogenicity of a Recombinant Adenovirus Serotype 26 HIV-1 Env Vaccine (IPCAVD 001). The Journal of infectious diseases 2012; 207(2): 240-7.

29. Kumar S, Maurya VK, Prasad AK, Bhatt MLB, Saxena SK. Structural, glycosylation and antigenic variation between 2019 novel coronavirus (2019-nCoV) and SARS coronavirus (SARS-CoV). Virusdisease 2020; 31(1): 13-21.

30. Wan Y, Shang J, Graham R, Baric RS, Li F. Receptor Recognition by the Novel Coronavirus from Wuhan: an Analysis Based on Decade-Long Structural Studies of SARS Coronavirus. Journal of virology 2020; 94(7).

31. Zost SJ, Parkhouse K, Gumina ME, et al. Contemporary H3N2 influenza viruses have a glycosylation site that alters binding of antibodies elicited by egg-adapted vaccine strains.

Proceedings of the National Academy of Sciences of the United States of America 2017; 114(47): 12578-83.

32. $\mathrm{Xu} \mathrm{H}$, Zhong L, Deng J, et al. High expression of ACE2 receptor of 2019-nCoV on the epithelial cells of oral mucosa. Int J Oral Sci 2020; 12(1): 8.

33. Aylward B, Liang W. Report of the WHO-China Joint Mission on Coronavirus Disease 2019 (COVID-19). WHO Report 2020.

34. Yeo C, Kaushal S, Yeo D. Enteric involvement of coronaviruses: is faecal-oral transmission of SARS-CoV-2 possible? Lancet Gastroenterol Hepatol 2020. 
35. Han C, Duan C, Zhang S, et al. Digestive Symptoms in COVID-19 Patients with Mild Disease Severity: Clinical Presentation, Stool Viral RNA Testing, and Outcomes. American Journal of Gastroenterology 2020.

36. Muramatsu M, Yoshida R, Yokoyama A, et al. Comparison of antiviral activity between IgA and IgG specific to influenza virus hemagglutinin: increased potential of IgA for heterosubtypic immunity. PLoS One 2014; 9(1): e85582.

37. Sterlin D, Mathian A, Miyara M, et al. IgA dominates the early neutralizing antibody response to SARS-CoV-2. medRxiv 2020: 2020.06.10.20126532.

38. Tamura S, Funato H, Hirabayashi Y, et al. Cross-protection against influenza A virus infection by passively transferred respiratory tract IgA antibodies to different hemagglutinin molecules. Eur J Immunol 1991; 21(6): 1337-44.

39. Le Bert N, Tan AT, Kunasegaran K, et al. SARS-CoV-2-specific T cell immunity in cases of COVID-19 and SARS, and uninfected controls. Nature 2020; 584(7821): 457-62.

40. Ni L, Ye F, Cheng ML, et al. Detection of SARS-CoV-2-Specific Humoral and Cellular Immunity in COVID-19 Convalescent Individuals. Immunity 2020; 52(6): 971-7 e3.

41. Tai W, Zhang X, Drelich A, et al. A novel receptor-binding domain (RBD)-based mRNA vaccine against SARS-CoV-2. Cell Res 2020.

42. Zha L, Zhao H, Mohsen MO, et al. Development of a COVID-19 vaccine based on the receptor binding domain displayed on virus-like particles. bioRxiv 2020: 2020.05.06.079830.

43. Mateus J, Grifoni A, Tarke A, et al. Selective and cross-reactive SARS-CoV-2 T cell epitopes in unexposed humans. Science 2020: eabd3871.

44. Mercado NB, Zahn R, Wegmann F, et al. Single-shot Ad26 vaccine protects against SARS-CoV-2 in rhesus macaques. Nature 2020.

45. Mutua G, Anzala O, Luhn K, et al. Safety and Immunogenicity of a 2-Dose Heterologous Vaccine Regimen With Ad26.ZEBOV and MVA-BN-Filo Ebola Vaccines: 12-Month Data From a Phase 1 Randomized Clinical Trial in Nairobi, Kenya. J Infect Dis 2019; 220(1): 57-67.

46. Anywaine Z, Whitworth H, Kaleebu P, et al. Safety and Immunogenicity of a 2-Dose Heterologous Vaccination Regimen With Ad26.ZEBOV and MVA-BN-Filo Ebola Vaccines: 12Month Data From a Phase 1 Randomized Clinical Trial in Uganda and Tanzania. J Infect Dis 2019; 220 (1): 46-56.

47. Antrobus RD, Coughlan L, Berthoud TK, et al. Clinical assessment of a novel recombinant simian adenovirus $\mathrm{ChAdOx} 1$ as a vectored vaccine expressing conserved Influenza A antigens. Mol Ther 2014; 22(3): 668-74.

48. van Doremalen N, Haddock E, Feldmann F, et al. A single dose of ChAdOx1 MERS provides broad protective immunity against a variety of MERS-CoV strains. bioRxiv 2020: 2020.04.13.036293.

49. Hassan AO, Kafai NM, Dmitriev IP, et al. A single intranasal dose of chimpanzee adenovirus-vectored vaccine confers sterilizing immunity against SARS-CoV-2 infection. bioRxiv 2020: 2020.07.16.205088.

50. He TC, Zhou S, da Costa LT, Yu J, Kinzler KW, Vogelstein B. A simplified system for generating recombinant adenoviruses. Proceedings of the National Academy of Sciences of the United States of America 1998; 95(5): 2509-14. 
51. Guthe S, Kapinos L, Moglich A, Meier S, Grzesiek S, Kiefhaber T. Very fast folding and association of a trimerization domain from bacteriophage T4 fibritin. J Mol Biol 2004; 337(4): 905-15.

52. Tucker SN, Lin K, Stevens S, Scollay R, Bennett MJ, Olson DC. Systemic and mucosal antibody responses following retroductal gene transfer to the salivary gland. Mol Therapy 2003; 8: $392-9$.

53. Tan CW, Chia WN, Qin X, et al. A SARS-CoV-2 surrogate virus neutralization test based on antibody-mediated blockage of ACE2-spike protein-protein interaction. Nat Biotechnol 2020 . 